\title{
Thermodynamic Analysis of the Nb-Ti-B Ternary Phase Diagram
}

\author{
Yu Nakama ${ }^{1}$, Hiroshi Ohtani ${ }^{2}$ and Mitsuhiro Hasebe ${ }^{2}$ \\ ${ }^{1}$ Graduate school of Engineering, Kyushu Institute of Technology, Kitakyushu 804-8550, Japan \\ ${ }^{2}$ Department of Materials Science, Faculty of Engineering, Kyushu Institute of Technology, Kitakyushu 804-8550, Japan
}

\begin{abstract}
A thermodynamic analysis of the $\mathrm{Nb}$ - Ti-B ternary system was carried out by estimating the unknown thermodynamic properties of binary and ternary borides by using a first-principles method. The calculated value for the binary $\mathrm{TiB}_{2}$ phase was in reasonable agreement with those found by experiment. Due to a lack of experimental values concerning thermodynamic properties of the ternary system, the formation enthalpies for the $\mathrm{Nb}_{3} \mathrm{~B}_{2}-\mathrm{Ti}_{3} \mathrm{~B}_{2}, \mathrm{NbB}-\mathrm{TiB}(\mathrm{Cmcm})$, NbB-TiB (Pnma), Nb5 $\mathrm{B}_{6}-\mathrm{Ti}_{5} \mathrm{~B}_{6}, \mathrm{Nb}_{3} \mathrm{~B}_{4}-\mathrm{Ti}_{3} \mathrm{~B}_{4}$, and $\mathrm{NbB}_{2}-\mathrm{TiB}_{2}$ pseudo-binary sections were also determined by constructing various kinds of superstructures for binary borides. The thermodynamic functions determined using these theoretical values, as well as the available experimental information on the phase fields, successfully revealed the phase equilibria in the $\mathrm{Nb}-\mathrm{Ti}-\mathrm{B}$ ternary system across the entire composition and all temperature ranges. [doi:10.2320/matertrans.MC200820]
\end{abstract}

(Received November 5, 2008; Accepted January 19, 2009; Published March 11, 2009)

Keywords: phase diagram, thermodynamic analysis, first-principles calculation, ternary borides

\section{Introduction}

The addition of small amounts of boron (B) to steels improves their strength and hardenability; therefore, this additive is used for various kinds of skinpass steel. This improvement in hardenability is generally explained by segregation of B on austenite grain boundaries, which suppresses the nucleation of ferrite on grain boundaries; however, this mechanism has not been clarified and is still controversial. On the other hand, the addition of $\mathrm{Nb}$ and $\mathrm{Ti}$ to steels improves their strength and grain refining by the formation of carbonitrides. Although the grain boundary segregation behavior of $\mathrm{B}$ is influenced by these additional elements, knowledge about the interaction with B is lacking. The objective of this study was to clarify the phase equilibria of the $\mathrm{Nb}-\mathrm{Ti}-\mathrm{B}$ ternary system across the entire range, using the Calculation of Phase Diagrams (CALPHAD) method. ${ }^{1)}$ Some thermodynamic properties of borides that were not available experimentally were evaluated by incorporating first-principles calculations.

\section{Computational Procedures}

This section discusses the computational procedures used in the first-principles calculations and the expression for the Gibbs free energy.

\subsection{First-principles calculations of the enthalpy of formation}

The enthalpy of formation of the boride phases was calculated using the full potential linearized augmented plane wave (FLAPW) method. ${ }^{2)}$ The FLAPW method, as implemented in the WIEN2k software package, ${ }^{3)}$ is one of the most accurate schemes for electronic calculations and allows precise calculations of the total energies in solids. Therefore, this method is most appropriate for the evaluation of relative thermodynamic properties, such as the formation enthalpy. The exchange-correlation functional was described by the generalized gradient approximation (GGA) of the PerdewBurke-Ernzerhof 96 form. ${ }^{4)}$ The plane-wave cutoff energy was set to $270 \mathrm{eV}$ throughout the calculation. For the integration we used a $12 \times 12 \times 12$ k-points mesh in the whole first Brillouin zone.

\subsection{Thermodynamic modeling}

\subsubsection{Liquid and terminal solution phases}

The regular solution approximation was applied to the liquid phase, and to the ( $\alpha \mathrm{Ti})$ and $\alpha$ solid solutions. The molar Gibbs energy, $G_{\mathrm{m}}^{\phi}$, was described as:

$$
\begin{aligned}
G_{\mathrm{m}}^{\phi}= & x_{\mathrm{Nb}}{ }^{\circ} G_{\mathrm{Nb}}^{\phi}+x_{\mathrm{Ti}}{ }^{\circ} G_{\mathrm{Ti}}^{\phi}+x_{\mathrm{B}}{ }^{\circ} G_{\mathrm{B}}^{\phi} \\
& +R T\left(x_{\mathrm{Nb}} \ln x_{\mathrm{Nb}}+x_{\mathrm{Ti}} \ln x_{\mathrm{Ti}}+x_{\mathrm{B}} \ln x_{\mathrm{B}}\right) \\
& +x_{\mathrm{Nb}} x_{\mathrm{Ti}} L_{\mathrm{Nb}, \mathrm{Ti}}^{\phi}+x_{\mathrm{Nb}} x_{\mathrm{B}} L_{\mathrm{Nb}, \mathrm{B}}^{\phi} \\
& +x_{\mathrm{Ti}} x_{\mathrm{B}} L_{\mathrm{Ti}, \mathrm{B}}^{\phi}+x_{\mathrm{Nb}} x_{\mathrm{Ti}} x_{\mathrm{B}} L_{\mathrm{Nb}, \mathrm{Ti}, \mathrm{B}}^{\phi},
\end{aligned}
$$

where ${ }^{\circ} G_{i}^{\phi}$ denotes the molar Gibbs energy of element $i$ in the $\phi$ state. This quantity, called the lattice stability parameter, is described by the following formula:

$$
\begin{aligned}
{ }^{\mathrm{o}} G_{i}^{\phi}-{ }^{\mathrm{o}} H_{i}^{\mathrm{ref}}= & A+B T+C T \ln T+D T^{2} \\
& +E T^{3}+F T^{7}+I T^{-1}+J T^{-9} .
\end{aligned}
$$

In the above equation, ${ }^{\circ} H_{i}^{\text {ref }}$ denotes the molar enthalpy of the pure element $i$ in its stable state at $T=298 \mathrm{~K}$. The parameter $L_{\mathrm{i}, \mathrm{j}}^{\phi}$ denotes the interaction energy between $i$ and $j$ in the $\phi$ phase, and has a compositional dependency following the Redlich-Kister polynomial ${ }^{5)}$ as:

$$
L_{\mathrm{i}, \mathrm{j}}^{\phi}={ }^{0} L_{\mathrm{i}, \mathrm{j}}^{\phi}+{ }^{1} L_{\mathrm{i}, \mathrm{j}}^{\phi}\left(x_{\mathrm{i}}-x_{\mathrm{j}}\right)+{ }^{2} L_{\mathrm{i}, \mathrm{j}}^{\phi}\left(x_{\mathrm{i}}-x_{\mathrm{j}}\right)^{2},
$$

where the temperature dependency was introduced as ${ }^{n} L_{\mathrm{i}, \mathrm{j}}^{\phi}=$ $\mathrm{a}+\mathrm{b} T$. The term $L_{\mathrm{Nb}, \mathrm{Ti}, \mathrm{B}}^{\varphi}$ is the ternary interaction parameter between the elements $\mathrm{Nb}, \mathrm{Ti}$, and $\mathrm{B}$.

\subsubsection{The $(\mathrm{Nb}, \mathrm{Ti}) \mathrm{B}_{2}$ phase}

The crystal structure of the $(\mathrm{Nb}, \mathrm{Ti}) \mathrm{B}_{2}$ phase was illustrated in Fig. 1, where the metallic atoms occupy one sublattice as shown by open circles, while B atoms do another sublattice given by black circles. Furthermore, in our recent analysis, ${ }^{6)}$ we adopted a two-sublattice model to the binary $\mathrm{NbB}_{2}$ phase, in which the $\mathrm{Nb}$ atoms and $\mathrm{B}$ atoms were substituted by vacancies in each sublattice, i.e., $\left(\mathrm{Nb}_{y_{\mathrm{Nb}}^{(1)}} \mathrm{Va}_{y_{\mathrm{Va}}^{(1)}}\right)_{1}\left(\mathrm{~B}_{y_{\mathrm{B}}^{(2)}} \mathrm{Va}_{y_{\mathrm{Va}}^{(2)}}\right)_{2}$. Therefore, for the ternary $(\mathrm{Nb}, \mathrm{Ti}) \mathrm{B}_{2}$ phase, the same type 


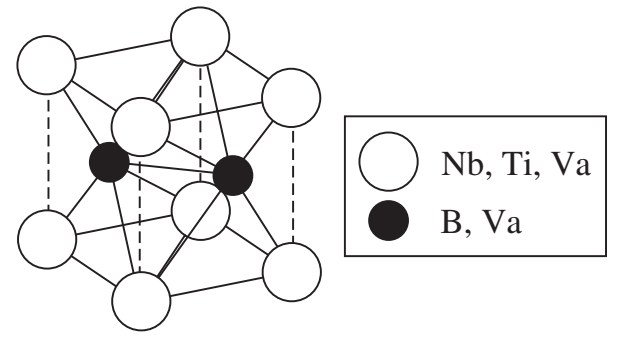

Fig. 1 Crystal structure of $(\mathrm{Nb}, \mathrm{Ti}) \mathrm{B}_{2}$ phase.

of two-sublattice model as $\left(\mathrm{Nb}_{y_{\mathrm{Nb}}^{(1)}} \mathrm{Ti}_{y_{\mathrm{Ti}}^{(1)}} \mathrm{Va}_{y_{\mathrm{Va}}^{(1)}}\right)_{1}\left(\mathrm{~B}_{y_{\mathrm{B}}^{(2)}} \mathrm{Va}_{y_{\mathrm{Va}}^{(2)}}\right)_{2}$ was employed, assuming that $\mathrm{Ti}$ atoms were substituted by $\mathrm{Nb}$ atoms in the first sublattice. This model reproduces the actual atomic configurations in the $(\mathrm{Nb}, \mathrm{Ti}) \mathrm{B}_{2}$ phase. The Gibbs energy per mole of formula unit of this phase is given by:

$$
\begin{aligned}
& G_{\mathrm{m}}^{(\mathrm{Nb}, \mathrm{Ti}) \mathrm{B}_{2}}=y_{\mathrm{Nb}}^{(1)}\left(y_{\mathrm{B}}^{(2) \circ} G_{\mathrm{Nb}: \mathrm{B}}^{(\mathrm{Nb}, \mathrm{Ti}) \mathrm{B}_{2}}+y_{\mathrm{Va}}^{(2) \circ} G_{\mathrm{Nb}: \mathrm{Va}}^{\left(\mathrm{Nb}, \mathrm{B}_{2}\right.}\right) \\
& +y_{\mathrm{Ti}}^{(1)}\left(y_{\mathrm{B}}^{(2) \circ} G_{\mathrm{Ti}: \mathrm{B}}^{(\mathrm{Nb}, \mathrm{Ti}) \mathrm{B}_{2}}+y_{\mathrm{Va}}^{(2) \circ} G_{\mathrm{Ti}: \mathrm{Va}}^{(\mathrm{Nb}, \mathrm{Ti}) \mathrm{B}_{2}}\right) \\
& +y_{\mathrm{Va}}^{(1)}\left(y_{\mathrm{B}}^{(2) \circ} G_{\mathrm{Va}: \mathrm{B}}^{(\mathrm{Nb}, \mathrm{Ti}) \mathrm{B}_{2}}+y_{\mathrm{Va}}^{(2) \circ} G_{\mathrm{Va}: \mathrm{Va}}^{\left(\mathrm{Nb}, \mathrm{B}_{2}\right.}\right) \\
& +0.333 R T\left(y_{\mathrm{Nb}}^{(1)} \ln y_{\mathrm{Nb}}^{(1)}+y_{\mathrm{Ti}}^{(1)} \ln y_{\mathrm{Ti}}^{(1)}+y_{\mathrm{Va}}^{(1)} \ln y_{\mathrm{Va}}^{(1)}\right) \\
& +0.667 R T\left(y_{\mathrm{B}}^{(2)} \ln y_{\mathrm{B}}^{(2)}+y_{\mathrm{Va}}^{(2)} \ln y_{\mathrm{Va}}^{(2)}\right) \\
& +y_{\mathrm{Nb}}^{(1)} y_{\mathrm{Ti}}^{(1)}\left(y_{\mathrm{B}}^{(2)} L_{\mathrm{Nb}, \mathrm{Ti}: \mathrm{B}}^{\left(\mathrm{Nb}, \mathrm{B}_{2}\right.}+y_{\mathrm{Va}}^{(2)} L_{\mathrm{Nb}, \mathrm{Ti}: \mathrm{Va}}^{\left(\mathrm{Nb}, \mathrm{B}_{2}\right.}\right) \\
& +y_{\mathrm{Nb}}^{(1)} y_{\mathrm{Va}}^{(1)}\left(y_{\mathrm{B}}^{(2)} L_{\mathrm{Nb}, \mathrm{Va}: \mathrm{B}}^{\left(\mathrm{Nb}, \mathrm{B}_{2}\right.}+y_{\mathrm{Va}}^{(2)} L_{\mathrm{Nb}, \mathrm{Va}: \mathrm{Va}}^{\left(\mathrm{Nb}, \mathrm{Ti} \mathrm{B}_{2}\right.}\right) \\
& +y_{\mathrm{Ti}}^{(1)} y_{\mathrm{Va}}^{(1)}\left(y_{\mathrm{B}}^{(2)} L_{\mathrm{Ti}, \mathrm{Va}: \mathrm{B}}^{\left(\mathrm{Nb}, \mathrm{Ti} \mathrm{B}_{2}\right.}+y_{\mathrm{Va}}^{(2)} L_{\mathrm{Ti}, \mathrm{Va}: \mathrm{Va}}^{(\mathrm{Nb}, \mathrm{Ti}) \mathrm{B}_{2}}\right) \\
& +y_{\mathrm{B}}^{(2)} y_{\mathrm{Va}}^{(2)}\left(y_{\mathrm{Nb}}^{(1)} L_{\mathrm{Nb}: \mathrm{B}, \mathrm{Va}}^{\left(\mathrm{Nb}, \mathrm{Ti} \mathrm{B}_{2}\right.}+y_{\mathrm{Ti}}^{(1)} L_{\mathrm{Ti}: \mathrm{B}, \mathrm{Va}}^{\left(\mathrm{Nb}, \mathrm{Ti} \mathrm{B}_{2}\right.}\right. \\
& +y_{\mathrm{Va}}^{(1)} L_{\mathrm{Va}: \mathrm{B}, \mathrm{Va}}^{(\mathrm{Nb}, \mathrm{Ti}) \mathrm{B}} \\
& +y_{\mathrm{Nb}}^{(1)} y_{\mathrm{Ti}}^{(1)} y_{\mathrm{Va}}^{(1)}\left(y_{\mathrm{B}}^{(2)} L_{\mathrm{Nb}, T \mathrm{Ti}, \mathrm{Va}: \mathrm{B}}^{\left(\mathrm{Nb}, \mathrm{B}_{2}\right.}+y_{\mathrm{Va}}^{(2)} L_{\mathrm{Nb}, \mathrm{Ti}, \mathrm{Va}: \mathrm{Va}}^{\left(\mathrm{Na}, \mathrm{Bi} \mathrm{B}_{2}\right.}\right) \\
& +y_{\mathrm{B}}^{(2)} y_{\mathrm{Va}}^{(2)}\left(y_{\mathrm{Nb}}^{(1)} y_{\mathrm{Ti}}^{(1)} L_{\mathrm{Nb}, \mathrm{Ti}: \mathrm{B}, \mathrm{Va}}^{\left(\mathrm{Nb}, \mathrm{Ti} \mathrm{B}_{2}\right.}+y_{\mathrm{Nb}}^{(1)} y_{\mathrm{Va}}^{(1)} L_{\mathrm{Nb}, \mathrm{Va}: \mathrm{B}, \mathrm{Va}}^{\left(\mathrm{Nb}, \mathrm{B}_{2}\right.}\right. \\
& \left.+y_{\mathrm{Ti}}^{(1)} y_{\mathrm{Va}}^{(1)} L_{\mathrm{Ti}, \mathrm{Va}: \mathrm{B}, \mathrm{Va}}^{\left(\mathrm{Ni}, \mathrm{Ti} \mathrm{B}_{2}\right.}\right) \\
& +y_{\mathrm{Nb}}^{(1)} y_{\mathrm{Ti}}^{(1)} y_{\mathrm{Va}}^{(1)} y_{\mathrm{B}}^{(2)} y_{\mathrm{Va}}^{(2)} L_{\mathrm{Nb}, \mathrm{Ti}, \mathrm{Va}: \mathrm{B}, \mathrm{Va}}
\end{aligned}
$$

where ${ }^{\circ} G_{\mathrm{Nb} \cdot \mathrm{B}}^{(\mathrm{Nb}) \mathrm{B}_{2}}$, for example, represents the Gibbs energy of a hypothetical compound, $\mathrm{Nb}_{1} \mathrm{~B}_{2}$, and terms relating to the same stoichiometry are identical. The parameter $L_{\mathrm{i}, \mathrm{j}: \mathrm{k}}^{(\mathrm{Nb}, \mathrm{Ti}) \mathrm{B}_{2}}$ denotes the interaction energy between unlike atoms on the first sublattice.

\subsubsection{The $\mathrm{Nb}_{3} \mathrm{~B}_{2}, \mathrm{NbB}, \mathrm{TiB}, \mathrm{Nb}_{5} \mathrm{~B}_{6}$, and $(\mathrm{Nb}, \mathrm{Ti})_{3} \mathrm{~B}_{4}$ phases}

The binary compounds, $\mathrm{Nb}_{3} \mathrm{~B}_{2}$, $\mathrm{NbB}$ ( Cmcm), TiB (Pnma), $\mathrm{Nb}_{5} \mathrm{~B}_{6}$, isostructural $\mathrm{Nb}_{3} \mathrm{~B}_{4}$ and $\mathrm{Ti}_{3} \mathrm{~B}_{4}$, dissolve the third metallic component to some extent, and therefore, the two-sublattice model denoted by $\left(\mathrm{Nb}_{y_{\mathrm{Nb}}^{(1)}}, \mathrm{Ti}_{y_{\mathrm{Ti}}^{(1)}}\right)_{m}\left(\mathrm{~B}_{y_{\mathrm{B}}^{(2)}=1}\right)_{n}$ was applied to these phases. The Gibbs energy per mole of formula unit of, for example, $(\mathrm{Nb}, \mathrm{Ti})_{3} \mathrm{~B}_{4}$ is given by:

$$
\begin{aligned}
G_{\mathrm{m}}^{(\mathrm{Nb}, \mathrm{Ti})_{3} \mathrm{~B}_{4}}= & y_{\mathrm{Nb}}^{(1) \circ} G_{\mathrm{Nb}: \mathrm{B}}^{(\mathrm{Nb}, \mathrm{Ti})_{3} \mathrm{~B}_{4}}+y_{\mathrm{Ti}}^{(1) \circ} G_{\mathrm{Ti}: \mathrm{B}}^{(\mathrm{Nb}, \mathrm{Ti})_{3} \mathrm{~B}_{4}} \\
& +0.429 R T\left(y_{\mathrm{Nb}}^{(1)} \ln y_{\mathrm{Nb}}^{(1)}+y_{\mathrm{Ti}}^{(1)} \ln y_{\mathrm{Ti}}^{(1)}\right) . \\
& +y_{\mathrm{Nb}}^{(1)} y_{\mathrm{Ti}}^{(1)} L_{\mathrm{Nb}, \mathrm{Ti}: \mathrm{B}}^{\left(\mathrm{Nb}, \mathrm{B}_{4}\right.}
\end{aligned}
$$

\subsubsection{Stoichiometric compounds}

The binary compound phase $\mathrm{Nb}_{2} \mathrm{~B}_{3}$ with a zero homogeneity range was treated as being a stoichiometric compound, and the Gibbs energy for the phase is described as in eq. (6):

$$
\begin{gathered}
{ }^{\circ} G_{\mathrm{Nb}: \mathrm{B}}^{\mathrm{Nb}_{2} \mathrm{~B}_{3}}-0.4^{\circ} G_{\mathrm{Nb}}^{\alpha}-0.6^{\circ} G_{\mathrm{B}}^{\beta \mathrm{B}} \\
=\mathrm{a}+\mathrm{b} T+\mathrm{c} T \ln T+\mathrm{d} T^{2} .
\end{gathered}
$$

\section{Thermodynamic Analysis and Calculation of the Phase Diagram}

A brief outline of the thermodynamic analysis and the calculated results are presented in this section. Most of the descriptions of lattice stability parameters for each pure element were obtained from the SGTE (Scientific Group Thermodata Europe) data set, ${ }^{7)}$ and are shown in Table 1.

\subsection{The Nb-Ti binary system}

The equilibrium phases of the $\mathrm{Nb}-\mathrm{Ti}$ binary system are the liquid (L) phase, a continuous series of bec solid solution $\alpha$, and the Ti terminal solution ( $\alpha \mathrm{Ti})$. Thermodynamic analysis of this binary system was performed by several researchers, ${ }^{8,9)}$ and agreement with experimental data is almost equal. The description by Kumar et al. ${ }^{10)}$ is accepted in the present work. The calculated $\mathrm{Nb}-\mathrm{Ti}$ binary phase diagram is shown in Fig. 2.

\subsection{The Nb-B binary system}

The equilibrium phase diagram of the $\mathrm{Nb}-\mathrm{B}$ binary system contains the liquid (L) phase, the terminal solutions of $\mathrm{Nb}(\alpha)$ and $\mathrm{B}(\beta \mathrm{B})$, and six intermetallic compounds: $\mathrm{Nb}_{3} \mathrm{~B}_{2}, \mathrm{NbB}$, $\mathrm{Nb}_{5} \mathrm{~B}_{6}, \mathrm{Nb}_{3} \mathrm{~B}_{4}, \mathrm{Nb}_{2} \mathrm{~B}_{3}$, and $\mathrm{NbB}_{2}$. The thermodynamic analysis concerning this binary system has already been done by our group. ${ }^{6)}$ However, to retain consistency with the Fe$\mathrm{Zr}$-B ternary system, ${ }^{11)}$ some thermodynamic parameters of the $\mathrm{NbB}_{2}$ phase were slightly modified. These parameters are summarized in Table 2, and the calculated phase diagram is compared with the experimental results in Fig. 3.

\subsection{The Ti-B binary system}

According to the critical assessment by Murray et al. ${ }^{12)}$ the equilibrium phase diagram of the Ti-B binary system contains the liquid $(\mathrm{L})$ phase, terminal solutions of Ti $((\alpha \mathrm{Ti})$ and $\alpha)$ and $\mathrm{B}(\beta \mathrm{B})$, and three intermetallic compounds, namely the $\mathrm{TiB}, \mathrm{Ti}_{3} \mathrm{~B}_{4}$, and $\mathrm{TiB}_{2}$ phases. The TiB phase has an orthorhombic FeB-type structure (Pnma), and its homogeneity range is narrow with a range of about 49 to $50 \mathrm{~mol} \%$ B. The structure of the diboride $\mathrm{TiB}_{2}$ is well established by several experimental studies, ${ }^{13-17)}$ having the $\mathrm{AlB}_{2}$ structure $(P 6 / m m m)$. The $\mathrm{Ti}_{3} \mathrm{~B}_{4}$ phase was absent from the phase diagram of Rudy et al. ${ }^{17)}$ which may be due to its narrow composition; however, Spear et al. ${ }^{18)}$ confirmed the existence of this phase through arc-melting and annealing studies. The crystal structure of $\mathrm{Ti}_{3} \mathrm{~B}_{4}$ was reported as isomorphous with that of $\mathrm{Nb}_{3} \mathrm{~B}_{4}($ Immm $),{ }^{16,18,19)}$ and the homogeneity range is rather small. The $\mathrm{Ti}_{3} \mathrm{~B}_{4}$ phase was said to transform to a hightemperature form at about $2283 \mathrm{~K}$, but the transformation is not reversible, and these allotropic forms were not considered in the present study.

The solubility of B in the $\alpha$ phase is less than $1 \mathrm{~mol} \%$ at the eutectic temperature $1813 \mathrm{~K},{ }^{17)}$ where solidification occurs by the eutectic reaction as $\mathrm{L} \rightleftarrows \alpha+\mathrm{TiB} .{ }^{17)}$ The peritectictype reaction between the $\alpha, \alpha \mathrm{Ti}$ and $\mathrm{TiB}$ phases occurs at around $1157 \mathrm{~K} .^{12,15,17)}$ The TiB phase forms from the melt by 
Table 1 Lattice stability parameters for $\mathrm{B}, \mathrm{Nb}$, and $\mathrm{Ti}$.

\begin{tabular}{|c|c|c|c|c|c|}
\hline Element & Phase & & Lattice stability parameter $(\mathrm{J} / \mathrm{mol})$ & Temperature (K) & Reference \\
\hline \multirow{9}{*}{ B } & \multirow{4}{*}{$\beta \mathrm{B}$} & \multirow{4}{*}{${ }^{\circ} G_{\mathrm{B}}^{\beta \mathrm{B}}-{ }^{\circ} H_{\mathrm{B}}^{\beta \mathrm{B}}$} & $\begin{aligned}= & -7735.284+107.111864 T-15.6641 T \ln T \\
& -6.864515 \times 10^{-3} T^{2}+6.18878 \times 10^{-7} T^{3}+370843 T^{-1}\end{aligned}$ & $298.15<T<1100$ & \multirow{9}{*}{ 7) } \\
\hline & & & $\begin{aligned}= & -16649.474+184.801744 T-26.6047 T \ln T \\
& -7.9809 \times 10^{-4} T^{2}-2.556 \times 10^{-8} T^{3}+1748270 T^{-1}\end{aligned}$ & $1100<T<2348$ & \\
\hline & & & $\begin{aligned}= & -36667.582+231.336244 T-31.5957527 T \ln T \\
& -1.59488 \times 10^{-3} T^{2}+1.34719 \times 10^{-7} T^{3}+11205883 T^{-1}\end{aligned}$ & $2348<T<3000$ & \\
\hline & & & $=-21530.653+222.396264 T-31.4 T \ln T$ & $3000<T<6000$ & \\
\hline & \multirow{3}{*}{$\mathrm{L}$} & \multirow{3}{*}{${ }^{\circ} G_{\mathrm{B}}^{\mathrm{L}}-{ }^{\circ} H_{\mathrm{B}}^{\beta \mathrm{B}}$} & $\begin{aligned}= & +40723.275+86.843839 T-15.6641 T \ln T \\
& -6.864515 \times 10^{-3} T^{2}+6.18878 \times 10^{-7} T^{3}+370843 T^{-1}\end{aligned}$ & $298.15<T<500$ & \\
\hline & & & $\begin{aligned}= & +41119.703+82.101722 T-14.9827763 T \ln T \\
& -7.095669 \times 10^{-3} T^{2}+5.07347 \times 10^{-7} T^{3}+335484 T^{-1}\end{aligned}$ & $500<T<2348$ & \\
\hline & & & $=+28842.012+200.94731 T-31.4 T \ln T$ & $2348<T<6000$ & \\
\hline & $\alpha$ & ${ }^{\circ} G_{\mathrm{B}}^{\alpha}-{ }^{\circ} G_{\mathrm{B}}^{\beta \mathrm{B}}$ & $=+43514-12.217 T$ & $298.15<T<6000$ & \\
\hline & $\alpha \mathrm{Ti}$ & ${ }^{\circ} G_{\mathrm{B}}^{\alpha \mathrm{Ti}}-{ }^{\circ} G_{\mathrm{B}}^{\beta \mathrm{B}}$ & $=+50208-9.706 T$ & $298.15<T<6000$ & \\
\hline \multirow{5}{*}{$\mathrm{Nb}$} & \multirow{2}{*}{$\alpha$} & \multirow{2}{*}{${ }^{\circ} G_{\mathrm{Nb}}^{\alpha}-{ }^{\circ} H_{\mathrm{Nb}}^{\alpha}$} & $\begin{aligned}= & -8519.353+142.045475 T-26.4711 T \ln T \\
& +2.03475 \times 10^{-4} T^{2}-3.5012 \times 10^{-7} T^{3}+93399 T^{-1}\end{aligned}$ & $298.15<T<2750$ & \multirow{5}{*}{ 7) } \\
\hline & & & $\begin{aligned}= & -37669.3+271.720843 T-41.77 T \ln T \\
& +1.528238 \times 10^{32} T^{-9}\end{aligned}$ & $2750<T<6000$ & \\
\hline & \multirow{2}{*}{$\mathrm{L}$} & \multirow{2}{*}{${ }^{\circ} G_{\mathrm{Nb}}^{\mathrm{L}}-{ }^{\circ} G_{\mathrm{Nb}}^{\alpha}$} & $=+29781.555-10.816418 T-3.06098 \times 10^{-23} T^{7}$ & $298.15<T<2750$ & \\
\hline & & & $=+30169.902-10.964695 T-1.528238 \times 10^{32} T^{-9}$ & $2750<T<6000$ & \\
\hline & $\alpha \mathrm{Ti}$ & ${ }^{\circ} G_{\mathrm{Nb}}^{\alpha \mathrm{Ti}}-{ }^{\circ} H_{\mathrm{Nb}}^{\alpha}$ & $\begin{aligned}= & +1480.65+144.448 T-26.4711 T \ln T \\
& +2.03475 \times 10^{-4} T^{2}-3.50119 \times 10^{-7} T^{3}+93398.8 T^{-1}\end{aligned}$ & $298.15<T<6000$ & \\
\hline \multirow{12}{*}{$\mathrm{Ti}$} & \multirow{4}{*}{$\alpha \mathrm{Ti}$} & \multirow{4}{*}{${ }^{\circ} G_{\mathrm{Ti}}^{\alpha \mathrm{Ti}}-{ }^{\circ} H_{\mathrm{Ti}}^{\alpha \mathrm{Ti}}$} & $\begin{aligned}= & -8059.921+133.615208 T-23.9933 T \ln T \\
& -4.777975 \times 10^{-3} T^{2}+1.06716 \times 10^{-7} T^{3}+72636 T^{-1}\end{aligned}$ & $298.15<T<900$ & \multirow{12}{*}{ 7) } \\
\hline & & & $\begin{aligned}= & -7811.815+132.988068 T-23.9887 T \ln T \\
& -4.2033 \times 10^{-3} T^{2}-9.0876 \times 10^{-8} T^{3}+42680 T^{-1}\end{aligned}$ & $900<T<1155$ & \\
\hline & & & $\begin{aligned}= & +908.837+66.976538 T-14.9466 T \ln T \\
& -8.1465 \times 10^{-3} T^{2}+2.02715 \times 10^{-7} T^{3}-1477660 T^{-1}\end{aligned}$ & $1155<T<1941$ & \\
\hline & & & $\begin{aligned}= & -124526.786+638.806871 T \\
& -87.2182461 T \ln T+8.204849 \times 10^{-3} T^{2} \\
& -3.04747 \times 10^{-7} T^{3}+36699805 T^{-1}\end{aligned}$ & $1941<T<4000$ & \\
\hline & \multirow{5}{*}{$\mathrm{L}$} & \multirow{5}{*}{${ }^{\circ} G_{\mathrm{Ti}}^{\mathrm{L}}-{ }^{\circ} H_{\mathrm{Ti}}^{\alpha \mathrm{Ti}}$} & $\begin{aligned}= & +4134.494+126.63427 T-23.9933 T \ln T \\
& -4.777975 \times 10^{-3} T^{2}+1.06716 \times 10^{-7} T^{3}+72636 T^{-1}\end{aligned}$ & $298.15<T<900$ & \\
\hline & & & $\begin{aligned}= & +4382.601+126.00713 T-23.9887 T \ln T \\
& -4.2033 \times 10^{-3} T^{2}-9.0876 \times 10^{-8} T^{3}+42680 T^{-1}\end{aligned}$ & $900<T<1155$ & \\
\hline & & & $\begin{aligned}= & +13103.253+59.9956 T-14.9466 T \ln T \\
& -8.1465 \times 10^{-3} T^{2}+2.02715 \times 10^{-7} T^{3}-1477660 T^{-1}\end{aligned}$ & $1155<T<1300$ & \\
\hline & & & $\begin{aligned}= & +369519.198-2556.0225 T-342.059267 T \ln T \\
& -0.163409355 T^{2}+1.2457117 \times 10^{-5} T^{3} \\
& -67034516 T^{-1}\end{aligned}$ & $1300<T<1941$ & \\
\hline & & & $=-19887.066+298.7367 T-46.29 T \ln T$ & $1941<T<4000$ & \\
\hline & \multirow{3}{*}{$\alpha$} & \multirow{3}{*}{${ }^{\circ} G_{\mathrm{Ti}}^{\alpha}-{ }^{\circ} H_{\mathrm{Ti}}^{\alpha \mathrm{Ti}}$} & $\begin{aligned}= & -1272.064+134.71418 T-25.5768 T \ln T \\
& -6.63845 \times 10^{-4} T^{2}-2.78803 \times 10^{-7} T^{3}+7208 T^{-1}\end{aligned}$ & $298.15<T<1155$ & \\
\hline & & & $\begin{aligned}= & +6667.385+105.366379 T-22.3771 T \ln T \\
& +1.21707 \times 10^{-3} T^{2}-8.4534 \times 10^{-7} T^{3}-2002750 T^{-1}\end{aligned}$ & $1155<T<1941$ & \\
\hline & & & $\begin{aligned}= & +26483.26-182.426471 T+19.0900905 T \ln T \\
& -2.200832 \times 10^{-2} T^{2}+1.228863 \times 10^{-6} T^{3}+1400501 T^{-1}\end{aligned}$ & $1941<T<4000$ & \\
\hline
\end{tabular}

a peritectic reaction with $\mathrm{Ti}_{3} \mathrm{~B}_{4}$ at $2453 \mathrm{~K}$. The peritectic reaction, $\mathrm{L}+\mathrm{TiB}_{2} \rightleftarrows \mathrm{Ti}_{3} \mathrm{~B}_{4}$, takes place at the slightly higher temperature of $2473 \mathrm{~K},{ }^{12}$ and the two peritectic reactions are separated by $20 \mathrm{~K}$. The $\mathrm{TiB}_{2}$ phase melts congruently; however, the experimental melting points showed some scattering in the range 3063-3498 K, possibly caused by vaporization. ${ }^{16)}$ Murray et al. ${ }^{12)}$ pointed out that the major difficulty of determining the melting temperature was due to the reaction with the crucible material, which results in the detection of a ternary eutectic temperature 


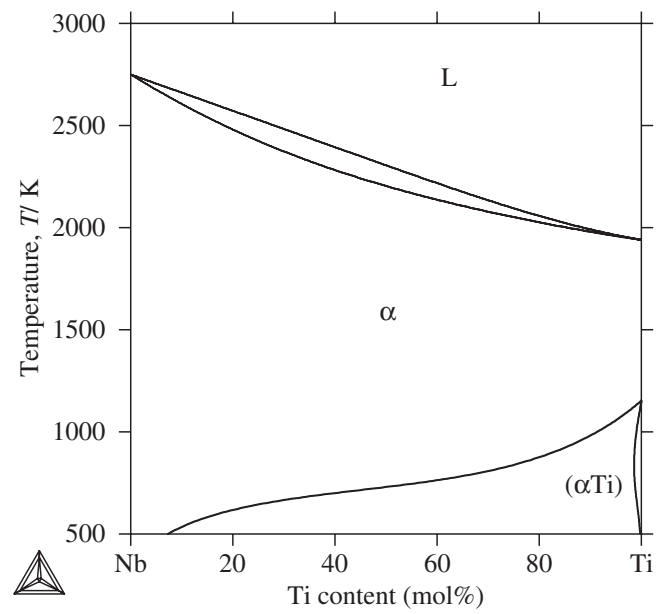

Fig. 2 The calculated Nb-Ti binary phase diagram.

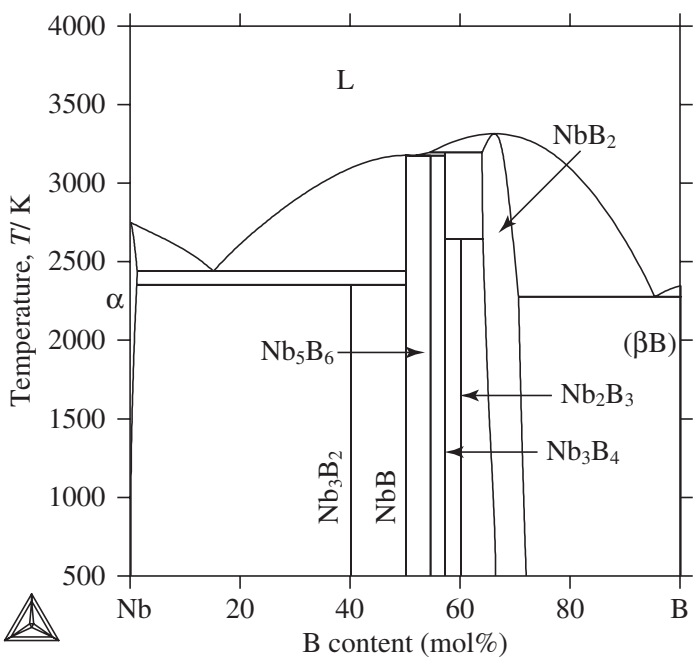

Fig. 3 The calculated Nb-B binary phase diagram.

Table 2 Optimized thermodynamic parameters for the binary and ternary systems.

\begin{tabular}{|c|c|c|c|}
\hline System & Phase & Thermodynamic parameter $(\mathrm{J} / \mathrm{mol})$ & Reference \\
\hline \multirow{3}{*}{$\mathrm{Nb}-\mathrm{Ti}$} & $\mathrm{L}$ & ${ }^{0} L_{\mathrm{Nb}, \mathrm{Ti}}^{\mathrm{L}}=+3000$ & \multirow{3}{*}{ 9) } \\
\hline & $\alpha$ & ${ }^{0} L_{\mathrm{Nb}, \mathrm{Ti}}^{\alpha}=+8900$ & \\
\hline & $\alpha \mathrm{Ti}$ & ${ }^{0} L_{\mathrm{Nb}, \mathrm{Ti}}^{\alpha \mathrm{Ti}}=+13150$ & \\
\hline \multirow{19}{*}{$\mathrm{Nb}-\mathrm{B}$} & \multirow{3}{*}{$\mathrm{L}$} & ${ }^{0} L_{\mathrm{Nb}, \mathrm{B}}^{\mathrm{L}}=-182310+5.02 T$ & \multirow{19}{*}{$\begin{array}{c}\text { Present } \\
\text { work }\end{array}$} \\
\hline & & ${ }^{1} L_{\mathrm{Nb}, \mathrm{B}}^{\mathrm{L}}=+13710-27.64 T$ & \\
\hline & & ${ }^{2} L_{\mathrm{Nb}, \mathrm{B}}^{\mathrm{L}}=+8840$ & \\
\hline & \multirow{3}{*}{$\alpha$} & ${ }^{0} L_{\mathrm{Nb}, \mathrm{B}}^{\alpha}=-85000+28.48 T$ & \\
\hline & & ${ }^{1} L_{\mathrm{Nb}, \mathrm{B}}^{\alpha}=-5000$ & \\
\hline & & ${ }^{2} L_{\mathrm{Nb}, \mathrm{B}}^{\alpha}=-68000$ & \\
\hline & $\mathrm{Nb}_{3} \mathrm{~B}_{2}$ & ${ }^{\circ} G_{\mathrm{Nb}: \mathrm{B}}^{\mathrm{Nb}_{3} \mathrm{~B}_{2}}-0.6^{\circ} G_{\mathrm{Nb}}^{\alpha}-0.4^{\circ} G_{\mathrm{B}}^{\beta \mathrm{B}}=-64430-12.81 T+2.02 T \ln T-3 \times 10^{-4} T^{2}$ & \\
\hline & $\mathrm{NbB}$ & ${ }^{\circ} G_{\mathrm{Nb}: \mathrm{B}}^{\mathrm{NbB}}-0.5^{\circ} G_{\mathrm{Nb}}^{\alpha}-0.5^{\circ} G_{\mathrm{B}}^{\beta \mathrm{B}}=-78710-11.01 T+1.7 T \ln T-9.8 \times 10^{-5} T^{2}$ & \\
\hline & $\mathrm{Nb}_{5} \mathrm{~B}_{6}$ & ${ }^{\circ} G_{\mathrm{Nb}: \mathrm{B}}^{\mathrm{Nb}_{5} \mathrm{~B}_{6}}-0.455^{\circ} G_{\mathrm{Nb}}^{\alpha}-0.545^{\circ} G_{\mathrm{B}}^{\beta \mathrm{B}}=-78740-14.33 T+2 T \ln T$ & \\
\hline & $\mathrm{Nb}_{3} \mathrm{~B}_{4}$ & ${ }^{\circ} G_{\mathrm{Nb}: \mathrm{B}}^{\mathrm{Nb}_{3} \mathrm{~B}_{4}}-0.429^{\circ} G_{\mathrm{Nb}}^{\alpha}-0.571^{\circ} G_{\mathrm{B}}^{\beta \mathrm{B}}=-78780-14.66 T+2 T \ln T$ & \\
\hline & $\mathrm{Nb}_{2} \mathrm{~B}_{3}$ & ${ }^{\circ} G_{\mathrm{Nb}: \mathrm{B}}^{\mathrm{Nb}_{2} \mathrm{~B}_{3}}-0.4^{\circ} G_{\mathrm{Nb}}^{\alpha}-0.6^{\circ} G_{\mathrm{B}}^{\beta \mathrm{B}}=-77350-15.33 T+2 T \ln T$ & \\
\hline & \multirow{8}{*}{$\mathrm{NbB}_{2}$} & ${ }^{\circ} G_{\mathrm{Nb}: \mathrm{B}}^{\mathrm{NbB}_{2}}-0.333^{\circ} G_{\mathrm{Nb}}^{\alpha}-0.667^{\circ} G_{\mathrm{B}}^{\beta B}=-72700+5.3 T-1.1 T \ln T+9 \times 10^{-4} T^{2}$ & \\
\hline & & ${ }^{\circ} G_{\mathrm{Nb}: \mathrm{Va}}^{\mathrm{Nb}_{2}}-0.333^{\circ} G_{\mathrm{Nb}}^{\alpha}=+19693$ & \\
\hline & & ${ }^{\circ} G_{\mathrm{Va:B}}^{\mathrm{NbB}_{2}}-0.667^{\circ} G_{\mathrm{B}}^{\beta \mathrm{B}}=+49554$ & \\
\hline & & ${ }^{\circ} G_{\mathrm{Va}: \mathrm{Va}_{2}}^{\mathrm{Nb}}=+100 T$ & \\
\hline & & ${ }^{0} L_{\mathrm{Nb}, \mathrm{Va}: \mathrm{B}}^{\mathrm{NbB}_{2}}=-84572+18 T$ & \\
\hline & & ${ }^{1} L_{\mathrm{Nb}, \mathrm{Va}: \mathrm{B}}^{\mathrm{NbB}}=-7644-10 T$ & \\
\hline & & ${ }^{0} L_{\mathrm{Nb}: \mathrm{B}, \mathrm{Va}}^{\mathrm{NbB}_{2}}=-80000+23 T$ & \\
\hline & & ${ }^{1} L_{\mathrm{Nb}: \mathrm{B}, \mathrm{Va}}^{\mathrm{Nb}}=+10000$ & \\
\hline
\end{tabular}

lower than the melting point of the binary boride. Thus, they employed the highest reported congruent temperature as $3498 \pm 25 \mathrm{~K}^{17)}$ in the assessed diagram.

Experimental thermodynamic data are available only for $\mathrm{TiB}_{2}$. The enthalpy of phase formation varies, ranging from -134 to $-324 \mathrm{~kJ} / \mathrm{mol}^{20-29)}$ The most recent paper by Yurick et al. ${ }^{30)}$ presented experimental measurements of equilibrium reactions of $\mathrm{TiB}_{2}$ with nitrogen, yielding the enthalpy of formation for $\mathrm{TiB}_{2}$ of $-304.2 \pm 3.8 \mathrm{~kJ} / \mathrm{mol}$ at $298 \mathrm{~K}$. The heat capacity values for the diboride have been measured in several studies. ${ }^{31-36)}$ Assessments of these data by Stull and Prophet ${ }^{27)}$ and Hultgren et al. ${ }^{28)}$ are in good agreement.

The thermodynamic analysis of this binary system was performed based on the experimental information above, as well as the calculated enthalpy of formation for the three types of $\mathrm{Ti}$ borides, using first-principles calculations. Information on the crystallographic data was obtained from Ref. 37). The calculated results are listed in Table 3. The listed values denote the enthalpy of formation based on hcp $\mathrm{Ti}$, and rhombohedral $\alpha \mathrm{B}$ with the hR12-type structure. 
Continued.

\begin{tabular}{|c|c|c|c|}
\hline System & Phase & Thermodynamic parameter $(\mathrm{J} / \mathrm{mol})$ & Reference \\
\hline \multirow{16}{*}{ Ti-B } & \multirow{4}{*}{$\mathrm{L}$} & ${ }^{0} L_{\mathrm{Ti}, \mathrm{B}}^{\mathrm{L}}=-265410+15.54 T$ & \multirow{16}{*}{$\begin{array}{l}\text { Present } \\
\text { work }\end{array}$} \\
\hline & & ${ }^{1} L_{\mathrm{Ti}, \mathrm{B}}^{\mathrm{L}}=-134300+17.71 T$ & \\
\hline & & ${ }^{2} L_{\mathrm{Nb}, \mathrm{B}}^{\mathrm{L}}=+61690$ & \\
\hline & & ${ }^{3} L_{\mathrm{Nb}, \mathrm{B}}^{\mathrm{L}}=+52660$ & \\
\hline & \multirow{2}{*}{$\alpha$} & ${ }^{0} L_{\mathrm{T}, \mathrm{B}}^{\alpha}=-75000+4 T$ & \\
\hline & & ${ }^{1} L_{\mathrm{Ti}, \mathrm{B}}^{\alpha}=+44000+2 T$ & \\
\hline & \multirow{2}{*}{$\alpha \mathrm{Ti}$} & ${ }^{0} L_{\mathrm{Ti}, \mathrm{B}}^{\alpha \mathrm{Ti}}=-80000$ & \\
\hline & & ${ }^{1} L_{\mathrm{Ti}, \mathrm{B}}^{\alpha \mathrm{Ti}}=+55000+3 T$ & \\
\hline & TiB & ${ }^{\circ} G_{\mathrm{Ti}: \mathrm{B}}^{\mathrm{TiB}}-0.5^{\circ} G_{\mathrm{Ti}}^{\alpha \mathrm{Ti}}-0.5^{\circ} G_{\mathrm{B}}^{\beta \mathrm{B}}=-83020+1.62 T$ & \\
\hline & $\mathrm{Ti}_{3} \mathrm{~B}_{4}$ & ${ }^{\circ} G_{\mathrm{Ti}: \mathrm{B}}^{\mathrm{Ti}_{3} \mathrm{~B}_{4}}-0.429^{\circ} G_{\mathrm{Ti}}^{\alpha \mathrm{Ti}}-0.571^{\circ} G_{\mathrm{B}}^{\beta \mathrm{B}}=-94390+3.05 T$ & \\
\hline & \multirow{6}{*}{$\mathrm{TiB}_{2}$} & $\begin{array}{l}{ }^{\circ} G_{\mathrm{Ti}: \mathrm{B}}^{\mathrm{TiB}_{2}}-0.333^{\circ} G_{\mathrm{Ti}}^{\alpha \mathrm{Ti}}-0.667^{\circ} G_{\mathrm{B}}^{\beta \mathrm{B}}=-105210+2.2 T-0.118 T \ln T \\
\quad+1.26 \times 10^{-3} T^{2}-9.71 \times 10^{-8} T^{3}+22089 T^{-1}\end{array}$ & \\
\hline & & ${ }^{\circ} G_{\mathrm{Ti}: \mathrm{Va}}^{\mathrm{TiB}_{2}}-0.333^{\circ} G_{\mathrm{Ti}}^{\alpha \mathrm{Ti}}=+11668$ & \\
\hline & & ${ }^{\circ} G_{\mathrm{Va}: \mathrm{B}}^{\mathrm{TiB}_{2}}-0.667^{\circ} G_{\mathrm{B}}^{\beta \mathrm{B}}=+49554$ & \\
\hline & & ${ }^{\circ} G_{\mathrm{Va}: \mathrm{Va}}^{\mathrm{TiB} 2}=+100 T$ & \\
\hline & & ${ }^{0} L_{\mathrm{Ti}, \mathrm{Va}: \mathrm{B}}^{\mathrm{TiB}_{2}}=-29300+T$ & \\
\hline & & ${ }^{0} L_{\mathrm{Ti} \mathrm{B}, \mathrm{Va}}^{\mathrm{Ti}_{2}}=+6610+2.5 T$ & \\
\hline \multirow{20}{*}{$\mathrm{Nb}-\mathrm{Ti}-\mathrm{B}$} & $\mathrm{L}$ & ${ }^{0} L_{\mathrm{Nb}, T \mathrm{Ti}, \mathrm{B}}^{\mathrm{L}}=+5.61 T$ & \multirow{20}{*}{$\begin{array}{l}\text { Present } \\
\text { work }\end{array}$} \\
\hline & & ${ }^{1} L_{\mathrm{Nb}, \mathrm{Ti}, \mathrm{B}}^{\mathrm{L}}=+187620-27 T$ & \\
\hline & & ${ }^{2} L_{\mathrm{Nb}, \mathrm{Ti}, \mathrm{B}}^{\mathrm{L}}=+152940+7.09 T$ & \\
\hline & & ${ }^{\circ} G_{\mathrm{Ti}: \mathrm{B}}^{\mathrm{Nb}_{3} \mathrm{~B}_{2}}-0.6^{\circ} G_{\mathrm{Ti}}^{\alpha \mathrm{Ti}}-0.4^{\circ} G_{\mathrm{B}}^{\beta \mathrm{B}}=-57760+7.21 T$ & \\
\hline & $\mathrm{Nb}_{3} \mathrm{~B}_{2}$ & ${ }^{0} L_{\mathrm{Nb}, \mathrm{Ti}: \mathrm{B}}^{\mathrm{Nb}_{3} \mathrm{~B}_{2}}=-13910+0.13 T$ & \\
\hline & & ${ }^{1} L_{\mathrm{Nb}, \mathrm{Ti}: \mathrm{B}}^{\mathrm{Nb}_{3}}=-9500-1.16 T$ & \\
\hline & \multirow{3}{*}{$\begin{array}{c}\mathrm{NbB} \\
(\mathrm{Cmcm})\end{array}$} & ${ }^{\circ} G_{\mathrm{Ti}: \mathrm{B}}^{\mathrm{NbB}}-0.5^{\circ} G_{\mathrm{Ti}}^{\alpha \mathrm{Ti}}-0.5^{\circ} G_{\mathrm{B}}^{\beta \mathrm{B}}=-82630+2.82 T$ & \\
\hline & & ${ }^{0} L_{\mathrm{Nb}, \mathrm{Ti}: \mathrm{B}}^{\mathrm{NbB}}=-5070+0.25 T$ & \\
\hline & & ${ }^{1} L_{\mathrm{Nb}, \mathrm{Ti}: \mathrm{B}}^{\mathrm{NbB}}=-2540-1.9 T$ & \\
\hline & \multirow{3}{*}{$\begin{array}{c}\mathrm{TiB} \\
(\text { Pnma })\end{array}$} & ${ }^{\circ} G_{\mathrm{Nb}: \mathrm{B}}^{\mathrm{TiB}}-0.5^{\circ} G_{\mathrm{Nb}}^{\alpha}-0.5^{\circ} G_{\mathrm{B}}^{\beta \mathrm{B}}=-78700+3.17 T$ & \\
\hline & & ${ }^{0} L_{\mathrm{Nb}, \mathrm{Ti}: \mathrm{B}}^{\mathrm{TiB}}=-7110-0.14 T$ & \\
\hline & & ${ }^{1} L_{\mathrm{Nb}, \mathrm{Ti}: \mathrm{B}}^{\mathrm{TiB}}=-2260-1.29 T$ & \\
\hline & \multirow{3}{*}{$\mathrm{Nb}_{5} \mathrm{~B}_{6}$} & ${ }^{\circ} G_{\mathrm{Ti}: \mathrm{B}}^{\mathrm{Nb} \mathrm{B}_{6}}-0.455^{\circ} G_{\mathrm{Ti}}^{\alpha \mathrm{Ti}}-0.545^{\circ} G_{\mathrm{B}}^{\beta \mathrm{B}}=-89030+5.19 T$ & \\
\hline & & ${ }^{0} L_{\mathrm{Nb}, T \mathrm{i}: \mathrm{B}}^{\mathrm{Nb}_{5} \mathrm{~B}_{6}}=-16000+4.15 T$ & \\
\hline & & ${ }^{1} L_{\mathrm{Nb}, \mathrm{Ti}: \mathrm{B}}^{\mathrm{Nb}_{6} \mathrm{~B}_{6}}=-8840-3.78 T$ & \\
\hline & \multirow{3}{*}{$(\mathrm{Nb}, \mathrm{Ti})_{3} \mathrm{~B}_{4}$} & ${ }^{0} L_{\mathrm{Nb}, \mathrm{Ti}: \mathrm{B}}^{\left(\mathrm{Nb}, \mathrm{B} \mathrm{B}_{4}\right.}=-15960+8.12 T$ & \\
\hline & & ${ }^{1} L_{\mathrm{Nb}, \mathrm{Ti}: \mathrm{B}}^{(\mathrm{Nb}, \mathrm{T})^{3} \mathrm{~B}_{4}}=-9810-7.74 T$ & \\
\hline & & ${ }^{2} L_{\mathrm{Nb}, \mathrm{Ti}: \mathrm{B}}^{(\mathrm{Nb}, \mathrm{T})_{4}}=+11000+1.97 T$ & \\
\hline & \multirow{2}{*}{$(\mathrm{Nb}, \mathrm{Ti}) \mathrm{B}_{2}$} & ${ }^{0} L_{\mathrm{Nb}, \mathrm{Ti}: \mathrm{B}}^{(\mathrm{Nb}, \mathrm{B}}{ }^{2}=-8570+3.21 T$ & \\
\hline & & ${ }^{1} L_{\mathrm{Nb}, \mathrm{Ti}: \mathrm{B}}^{(\mathrm{Nb}, \mathrm{B} 2}=+1610$ & \\
\hline
\end{tabular}

The formation energy calculated for the $\mathrm{Ti}_{3} \mathrm{~B}_{4}$ phase by the first-principles calculation did not make this boride stable at absolute zero temperature, and the value was then evaluated using the CALPHAD method. The calculated enthalpy of formation of $\mathrm{TiB}_{2}$ has been determined to be -315.6 $\mathrm{kJ} / \mathrm{mol}$, which agrees well with the experimental value of $-304.2 \pm 3.8 \mathrm{~kJ} / \mathrm{mol}$ at $298 \mathrm{~K}^{30)}$ In addition, the structure of the diboride $\mathrm{TiB}_{2}$ is isomorphous with the $\mathrm{NbB}_{2}$ phase with a large homogeneity range, extending from 61 to $70 \mathrm{~mol} \% \mathrm{~B}$. We carried out a ground state analysis on the $\mathrm{TiB}_{2}$ phase by constructing superstructures based on the $P 6 /$ mmm space group, ${ }^{6}$ as given in Table 3 . The calculated results are shown in Fig. 4. Thus, the thermodynamic parameters required for the $\left(\mathrm{Ti}_{y_{\mathrm{T}}^{(1)}} \mathrm{Va}_{y_{\mathrm{V}}^{(1)}}\right)_{1}\left(\mathrm{~B}_{y_{\mathrm{R}}^{(2)}} \mathrm{Va}_{y_{\mathrm{V}}^{(2)}}\right)_{2}$-type sublattice model were evaluated based on these results, as shown by the solid line in Fig. 4. Moreover, the heat capacity data at constant pressure by Chase $^{38)}$ were also considered in the thermodynamic analysis to evaluate the temperature dependency of the formation energy for the stoichiometric $\mathrm{TiB}_{2}$. The calculated values for the heat capacity were compared with the experimental data in Fig. 5. Figure 6 shows a comparison of the calculated and experimental data of the Ti-B binary phase diagram by Rudy et al. ${ }^{17)}$ It can be seen that the evaluated parameters show a good agreement with the corresponding experimental data on the phase boundaries. 
Table 3 Calculated enthalpy of formation for the compounds at the ground state in the Ti-B binary system.

\begin{tabular}{|c|c|c|c|c|}
\hline \multirow{2}{*}{ Phase } & \multirow{2}{*}{ Space group } & \multirow{2}{*}{$\begin{array}{l}\text { Composition } \\
\text { (mol\% B) }\end{array}$} & \multicolumn{2}{|c|}{ Enthalpy of formation $(\mathrm{kJ} / \mathrm{mol})$} \\
\hline & & & First principles & Assessed \\
\hline TiB & Pnma & 50.0 & -83.0 & $-82.5^{45)}$ \\
\hline $\mathrm{Ti}_{3} \mathrm{~B}_{4}$ & Immm & 57.1 & -85.5 & $-94.3^{45)}$ \\
\hline \multirow{11}{*}{$\mathrm{TiB}_{2}$} & $P 6 / \mathrm{mmm}$ & 0.0 & +35.0 & - \\
\hline & $\operatorname{Imm} 2$ & 33.3 & -32.1 & - \\
\hline & $P 6 / \mathrm{mmm}$ & 50.0 & -68.2 & - \\
\hline & $P 6 / \mathrm{mmm}$ & 57.1 & -83.1 & - \\
\hline & P6/mmm & 66.7 & -105.2 & $-109.7^{45)}$ \\
\hline & $P 6 / \mathrm{mmm}$ & 72.7 & -78.9 & - \\
\hline & P6/mmm & 75.0 & -68.7 & - \\
\hline & P6/mmm & 77.8 & -56.6 & - \\
\hline & P6/mmm & 80.0 & -46.4 & - \\
\hline & Fmmm & 84.2 & -21.7 & - \\
\hline & $P 6 / \mathrm{mmm}$ & 100.0 & +68.1 & - \\
\hline
\end{tabular}

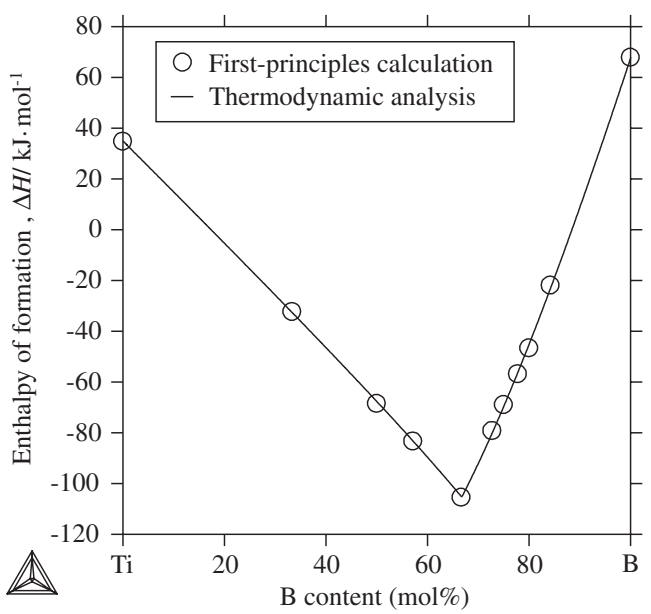

Fig. 4 Variation of the enthalpy of formation of $\mathrm{TiB}_{2}$ with composition.

\subsection{The Nb-Ti-B ternary system}

Concerning the $\mathrm{Nb}-\mathrm{Ti}-\mathrm{B}$ ternary system, there is some experimental information on phase equilibria by Kuz' ma ${ }^{39)}$ and Borisov et al. ${ }^{40)}$ A thermodynamic analysis of the entire ternary system was carried out by Witusiewicz et al. ${ }^{41}$ )

Kuz'ma ${ }^{39)}$ reported an isothermal section at $1673 \mathrm{~K}$ examined using the $\mathrm{X}$-ray diffraction method. The isothermal section is composed of the $\mathrm{Nb}_{3} \mathrm{~B}_{2}, \mathrm{NbB}, \mathrm{TiB}, \mathrm{Nb}_{3} \mathrm{~B}_{4}$, and $(\mathrm{Nb}, \mathrm{Ti}) \mathrm{B}_{2}$ phases, and no ternary compound exists. The solubility of $\mathrm{Ti}$ in the $\mathrm{Nb}_{3} \mathrm{~B}_{4}$ phase attains $5 \mathrm{~mol} \%$, and $\mathrm{TiB}$ dissolves up to $5 \mathrm{~mol} \% \mathrm{Nb}$. The solubility of $\mathrm{Ti}$ in $\mathrm{Nb}_{3} \mathrm{~B}_{2}$ is also small at less than $5 \mathrm{~mol} \%$. In contrast, the $\mathrm{NbB}$ phase dissolves up to $30 \mathrm{~mol} \%$ Ti. The isostructural borides $\mathrm{NbB}_{2}$ and $\mathrm{TiB}_{2}$ form a continuous solid solution $(\mathrm{Nb}, \mathrm{Ti}) \mathrm{B}_{2}$ in this ternary system. Borisov et al. ${ }^{40)}$ also examined a partial isothermal section diagram at $1673 \mathrm{~K}$, a vertical section at $7.5 \mathrm{~mol} \% \mathrm{~B}$, and projections of the solidus and liquidus surfaces with X-ray diffraction, metallography, electron probe microanalysis (EPMA), and differential thermal analysis. They showed that NbB $(\mathrm{Cmcm})$ and TiB (Pnma) yield a

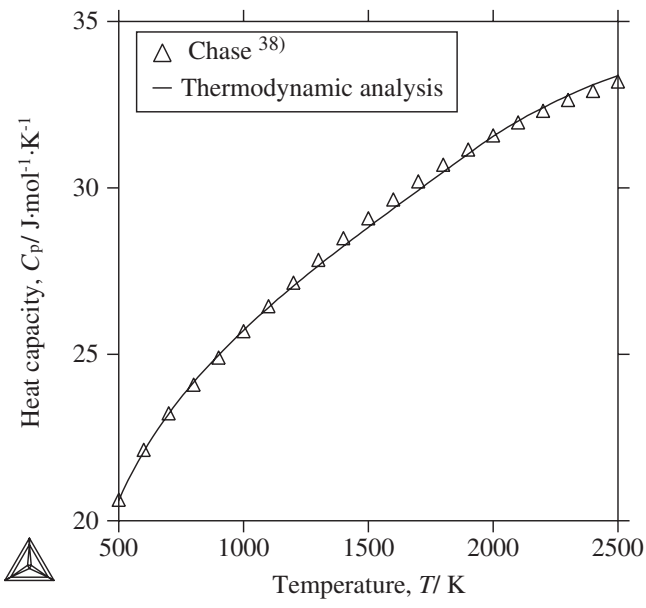

Fig. 5 Heat capacity of $\mathrm{TiB}_{2}$ in the Ti-B system.

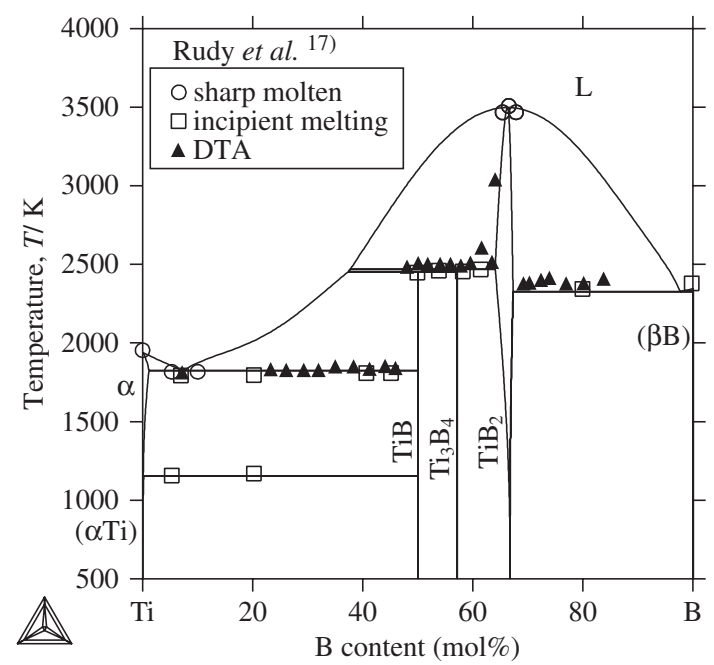

Fig. 6 The calculated Ti-B binary phase diagram.

narrow three-phase triangle for the $\alpha, \mathrm{NbB}$, and TiB phases, which is due to a difference in structure between the $\mathrm{Nb}$ - and Ti-based monoboride phases. According to the measurement of the $\mathrm{Nb}$ partition using EPMA, the mutual solubility values of $\mathrm{Nb}$ in $\mathrm{TiB}$ and of $\mathrm{Ti}$ in $\mathrm{NbB}$ are no less than 23 and $15 \mathrm{~mol} \%$, respectively. The solubility of $\mathrm{Ti}$ in $\mathrm{Nb}_{3} \mathrm{~B}_{4}$ is approximately $7 \mathrm{~mol} \%$, whereas that of $\mathrm{Nb}$ in $\mathrm{Ti}_{3} \mathrm{~B}_{4}$ is approximately $2 \mathrm{~mol} \%{ }^{40)}$ These results are somewhat different from those by Kuz'ma; however, the former data might be more accurate and preferable for optimization, because the solubility values were checked using several different experimental methods. The solidus and liquidus projections using EPMA and microstructure analysis were also constructed in the experimental study.

To cover the lack of the experimental values concerning the thermodynamic properties of the $\mathrm{Nb}-\mathrm{Ti}-\mathrm{B}$ ternary system, variation of formation energies for the binary borides with dissolving third component was evaluated in the present study using first-principles calculations. Superstructures for computing the formation energies were constructed by changing the stack of atoms along a given direction of the parent structure lattices. ${ }^{6,42,43)}$ Sixty types of superstructure for the $\mathrm{Nb}_{3} \mathrm{~B}_{2}-\mathrm{Ti}_{3} \mathrm{~B}_{2}$, for example, were used during the 
analysis and the nine structures having the lowest energies at the corresponding compositions were selected, as given in Table 4. Volume optimization was performed by varying total volume with constant axes ratio of a superstructure, and the total energy was expressed as a function of the volume using the Murnaghan equation of state. ${ }^{44)}$ The corresponding results are summarized in Table 4 for the $\mathrm{Nb}_{3} \mathrm{~B}_{2}-\mathrm{Ti}_{3} \mathrm{~B}_{2}$, $\mathrm{NbB}-\mathrm{TiB} \quad(\mathrm{Cmcm}), \quad \mathrm{NbB}-\mathrm{TiB} \quad$ (Pnma), $\quad \mathrm{Nb}_{5} \mathrm{~B}_{6}-\mathrm{Ti}_{5} \mathrm{~B}_{6}$, $\mathrm{Nb}_{3} \mathrm{~B}_{4}-\mathrm{Ti}_{3} \mathrm{~B}_{4}$, and $\mathrm{NbB}_{2}-\mathrm{TiB}_{2}$ pseudo-binary sections. These calculated values were used as a kind of experimental information to evaluate our thermodynamic parameters by fitting eqs. (4) and (5).

Our thermodynamic analysis was performed using the abovementioned experimental phase diagrams of Kuz'ma ${ }^{39}$ ) and Borisov et al., ${ }^{40)}$ and the energies of formation of the six pseudo-binary sections determined using first-principles calculations. In addition to the information on the solid phases, the experimental liquidus surface was also considered to estimate the ternary interaction parameters in the melt. The optimized thermodynamic parameters are summarized in Table 2. In Fig. 7(a) to (f), the calculated energies of formation of the six pseudo-binary sections were compared with those obtained using the conventional CALPHAD approach $^{41)}$ depicted by the dashed lines. The solid lines in these figures represent the results obtained by the present thermodynamic analysis. We can observe considerable difference between the calculated values and those obtained using the conventional CALPHAD approach. A shortcoming of the CALPHAD approach is that it is hard to obtain information on metastable equilibria, because the thermodynamic parameters from this method can only be evaluated from experimental data. Thus, the deduced thermodynamic behavior of the pseudo-binary borides in their metastable regions is quite controversial, mainly because of difficulties in experimental verification. Such serious problems, which are intrinsic to the CALPHAD approach, can be solved with the assistance of the first-principles energy calculations. From this viewpoint, the first-principles values are regarded more reliable than those obtained by the conventional CALPHAD method. Figure 8 shows the calculated $\mathrm{Nb}$ partition between the matrix phase and the ternary monoboride phases. The Nb content in the phases, as well as the directions and positions of the tie-lines, agree satisfactorily well with the experimental data obtained by Borisov et al. ${ }^{40)}$ The calculated isothermal sections at $1673 \mathrm{~K}$ are shown in Fig. 9(a) and (b), in comparison with the data obtained by Kuz'ma ${ }^{39)}$ and Borisov et al. ${ }^{40)}$ respectively. The calculated vertical section for the alloys at 7.5 at $\%$ B is shown in Fig. 10. Here the agreement is also satisfactory. Figure 11 shows the projections of the liquidus surface, and the details of the five invariant reactions are summarized in Table 5, comparing the data with the experimental work ${ }^{40)}$ and the previous calculation. ${ }^{41)}$

\section{Conclusions}

A thermodynamic analysis of the $\mathrm{Nb}-\mathrm{Ti}-\mathrm{B}$ ternary system was performed by incorporating first-principles calculations into the CALPHAD approach, yielding the following results.
Table 4 Calculated enthalpy of formation for the compounds at the ground state in the $\mathrm{Nb}-\mathrm{Ti}-\mathrm{B}$ ternary system.

\begin{tabular}{|c|c|c|c|c|}
\hline \multirow{2}{*}{ Phase } & \multirow{2}{*}{ Space group } & \multirow{2}{*}{$\begin{array}{c}\text { Site fraction } \\
\text { of } \mathrm{Ti}\end{array}$} & \multicolumn{2}{|c|}{ Enthalpy of formation $(\mathrm{kJ} / \mathrm{mol})$} \\
\hline & & & First principles & Assessed \\
\hline \multirow{9}{*}{$(\mathrm{Nb}, \mathrm{Ti})_{3} \mathrm{~B}_{2}$} & $P 4 / m b m$ & 0.000 & -64.4 & $-74.5^{45)}$ \\
\hline & $I 4 / m$ & 0.083 & -65.8 & - \\
\hline & Imma & 0.167 & -66.6 & - \\
\hline & $I 4 / m$ & 0.250 & -66.8 & - \\
\hline & $P 4 / m b m$ & 0.333 & -66.7 & - \\
\hline & Amm2 & 0.417 & -65.7 & - \\
\hline & Amm2 & 0.500 & -64.5 & - \\
\hline & $C 2 / m$ & 0.667 & -62.7 & - \\
\hline & $P 4 / m b m$ & 1.000 & -57.8 & $-60.0^{41)}$ \\
\hline \multirow{7}{*}{$\mathrm{NbB}$} & Cmcm & 0.000 & -78.7 & $-78.7^{45)}$ \\
\hline & $C 2$ & 0.125 & -79.2 & - \\
\hline & $C 2$ & 0.167 & -79.4 & - \\
\hline & $C 2 / m$ & 0.500 & -82.2 & - \\
\hline & $\mathrm{Cmcm}$ & 0.667 & -82.4 & - \\
\hline & Amm 2 & 0.833 & -82.6 & - \\
\hline & $\mathrm{Cmcm}$ & 1.000 & -82.6 & $-67.9^{41)}$ \\
\hline \multirow{7}{*}{ TiB } & Pnma & 0.000 & -78.7 & $-69.3^{41)}$ \\
\hline & $\mathrm{Cm}$ & 0.125 & -80.1 & - \\
\hline & Pnma & 0.333 & -82.0 & - \\
\hline & $P 2_{1} / c$ & 0.500 & -82.7 & - \\
\hline & Pnma & 0.667 & -82.9 & - \\
\hline & $P m$ & 0.875 & -83.0 & - \\
\hline & Pnma & 1.000 & -83.0 & $-82.5^{45)}$ \\
\hline \multirow{7}{*}{$(\mathrm{Nb}, \mathrm{Ti})_{5} \mathrm{~B}_{6}$} & Cmmm & 0.000 & -78.7 & $-80.6^{45)}$ \\
\hline & Immm & 0.100 & -82.2 & - \\
\hline & Cmmm & 0.200 & -84.9 & - \\
\hline & Amm2 & 0.300 & -86.2 & - \\
\hline & Amm2 & 0.600 & -88.2 & - \\
\hline & Amm2 & 0.800 & -88.8 & - \\
\hline & Cmmm & 1.000 & -89.0 & $+2.3^{41)}$ \\
\hline \multirow{7}{*}{$(\mathrm{Nb}, \mathrm{Ti})_{3} \mathrm{~B}_{4}$} & Immm & 0.000 & -78.8 & $-81.7^{45)}$ \\
\hline & $C 2 / m$ & 0.222 & -85.5 & - \\
\hline & Immm & 0.333 & -88.7 & - \\
\hline & $\operatorname{Imm} 2$ & 0.444 & -90.6 & - \\
\hline & $C 2 / m$ & 0.556 & -90.8 & - \\
\hline & Immm & 0.778 & -92.5 & - \\
\hline & Immm & 1.000 & -85.5 & $-94.3^{45)}$ \\
\hline \multirow{7}{*}{$(\mathrm{Nb}, \mathrm{Ti}) \mathrm{B}_{2}$} & $P 6 / \mathrm{mmm}$ & 0.000 & -72.7 & $-84.4^{45)}$ \\
\hline & Cmmm & 0.200 & -80.1 & - \\
\hline & $R \overline{3} m$ & 0.333 & -85.3 & - \\
\hline & $P 6 / \mathrm{mmm}$ & 0.500 & -90.9 & - \\
\hline & $\mathrm{P6} / \mathrm{mmm}$ & 0.667 & -96.2 & - \\
\hline & $\mathrm{P6} / \mathrm{mmm}$ & 0.833 & -101.1 & - \\
\hline & $\mathrm{P6} / \mathrm{mmm}$ & 1.000 & -105.2 & $-109.7^{45)}$ \\
\hline
\end{tabular}

(1) The thermodynamic analysis of the Ti-B binary system was carried out based on experimental information concerning phase boundaries and thermody- 


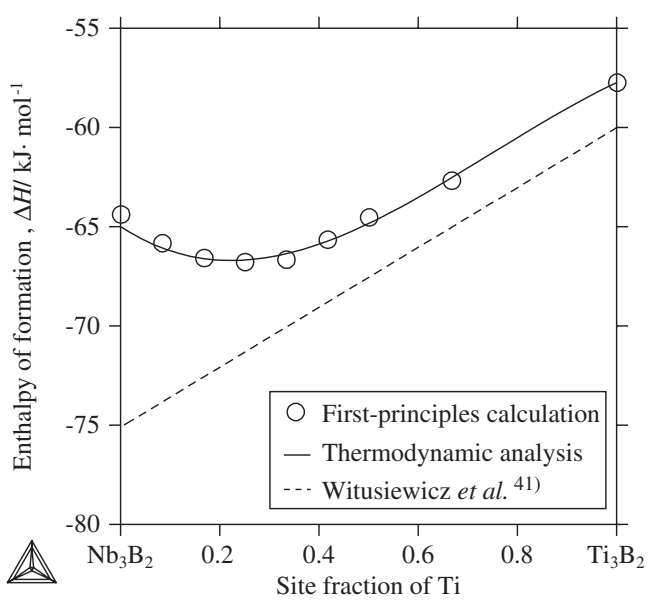

(a)

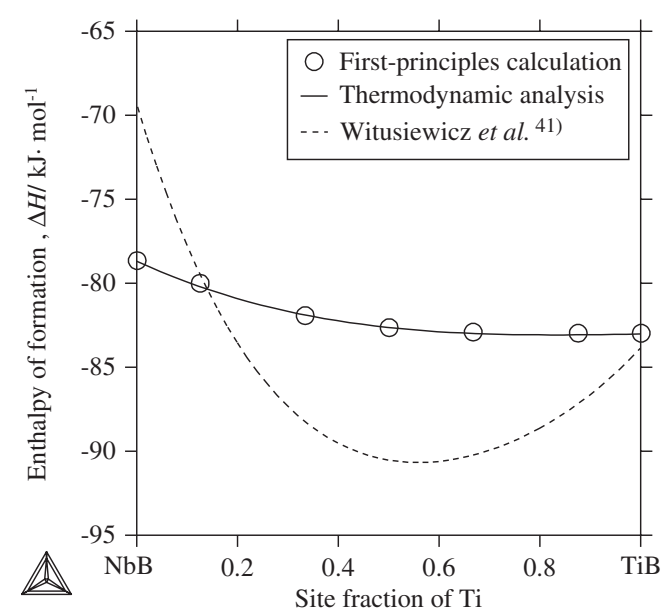

(c)

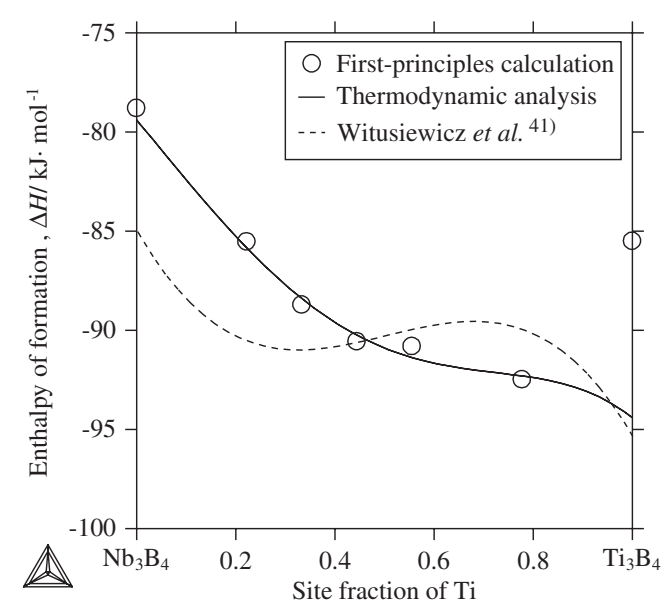

(e)

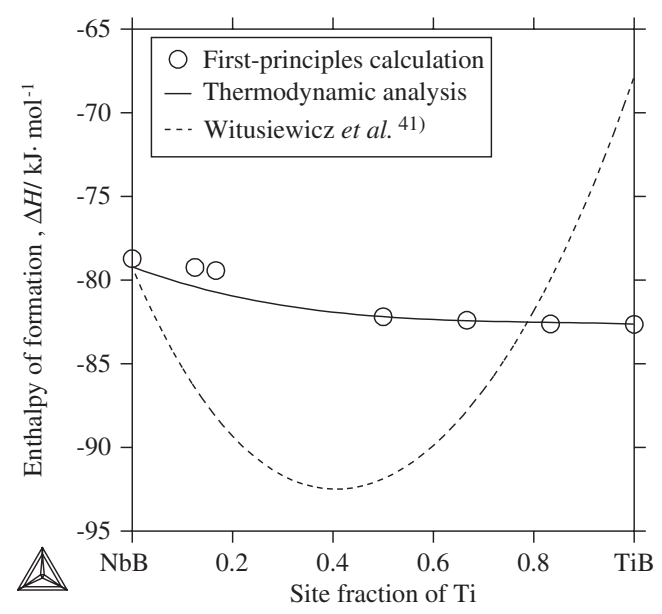

(b)

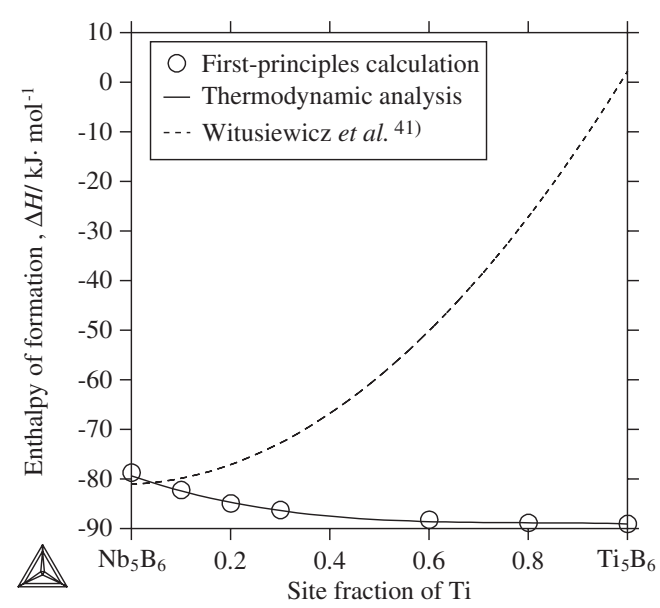

(d)

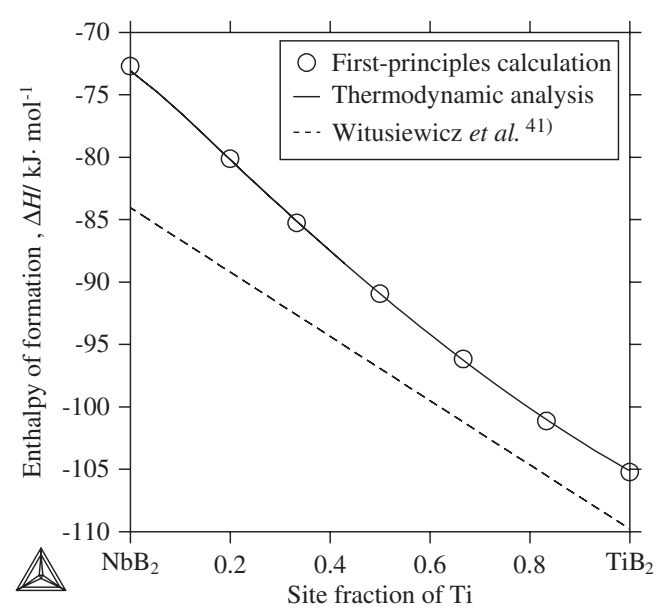

(f)

Fig. 7 Variation of the enthalpy of formation of (a) $\mathrm{Nb}_{3} \mathrm{~B}_{2}$, (b) $\mathrm{NbB}$ (c) $\mathrm{TiB}$ (d) $\mathrm{Nb}_{5} \mathrm{~B}_{6}$ (e) (Nb,Ti) ( $_{4}$ (f) (Nb,Ti) $\mathrm{B}_{2}$ with composition.

namic properties, as well as the calculated enthalpy of formation for the $\mathrm{TiB}, \mathrm{Ti}_{3} \mathrm{~B}_{4}$, and $\mathrm{TiB}_{2}$ phases, using first-principles calculations. The calculated enthalpy of formation of $\mathrm{TiB}_{2}$ was $-315.6 \mathrm{~kJ} / \mathrm{mol}$, which agrees well with the corresponding experimental value.
However, the formation energy of the $\mathrm{Ti}_{3} \mathrm{~B}_{4}$ phase calculated using first-principles calculations did not make this boride stable at absolute zero temperature, and the value was then evaluated using the CALPHAD method. 


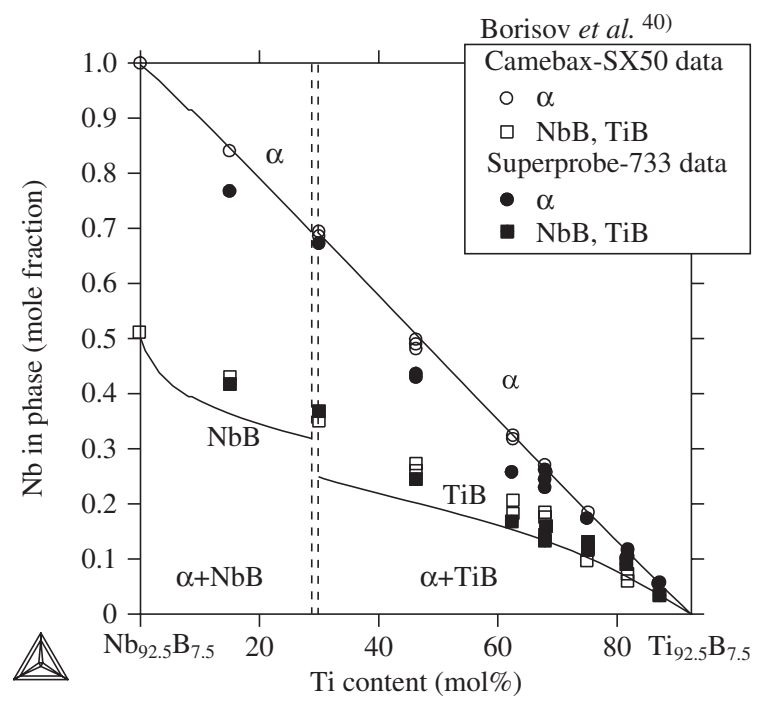

Fig. $8 \mathrm{Nb}$ content in the $\alpha, \mathrm{TiB}$ and $\mathrm{NbB}$ phases.

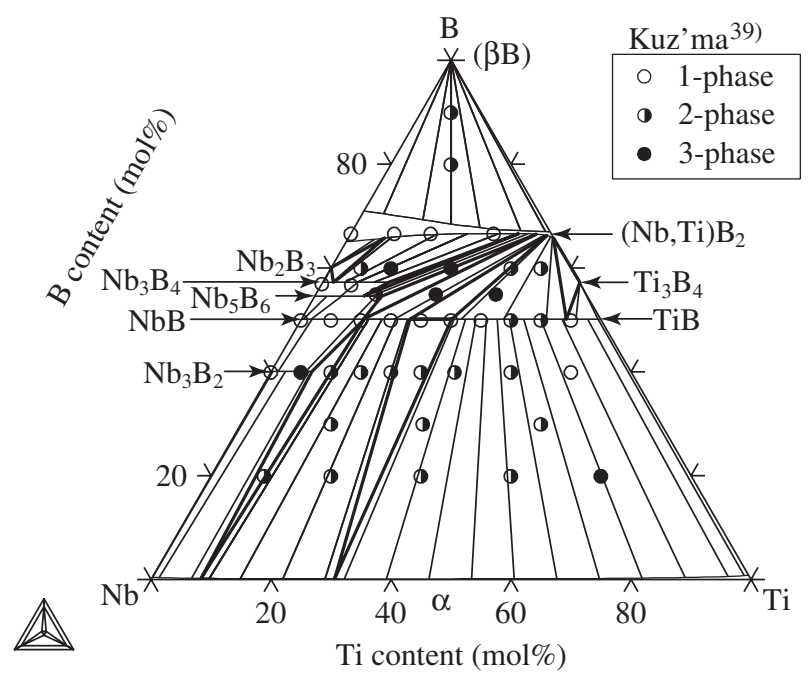

(a)

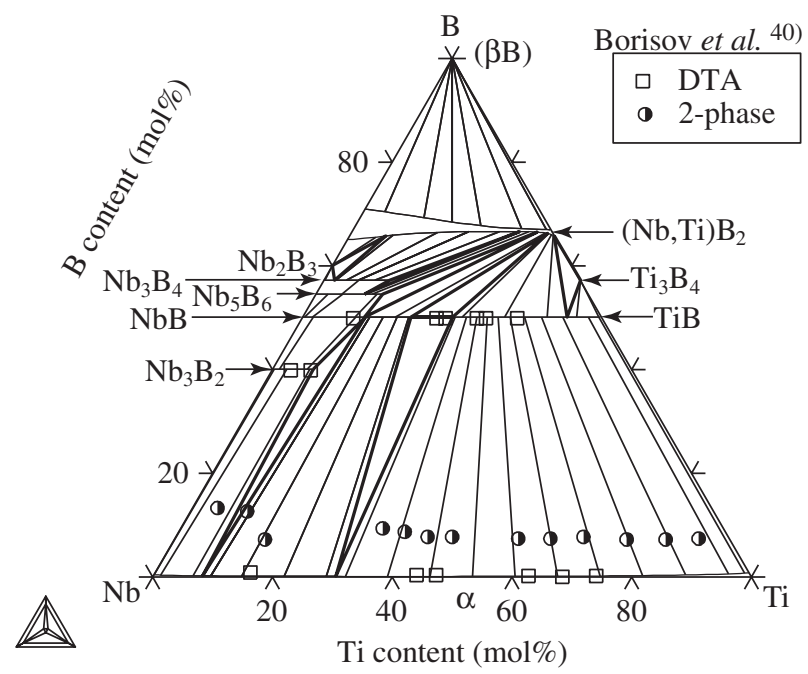

(b)

Fig. 9 Calculated isothermal section diagrams of the $\mathrm{Nb}-\mathrm{Ti}-\mathrm{B}$ system at $1673 \mathrm{~K}$ compared (a) with Kuz'ma; (b) with Borisov et al.

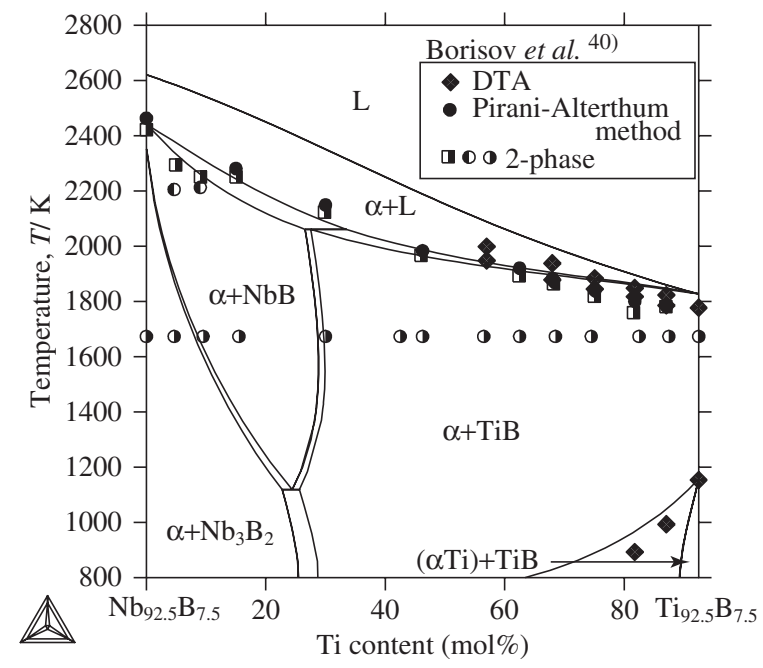

Fig. 10 The calculated vertical at $7.5 \mathrm{~mol} \% \mathrm{~B}$ for the $\mathrm{Nb}-\mathrm{Ti}-\mathrm{B}$ system.

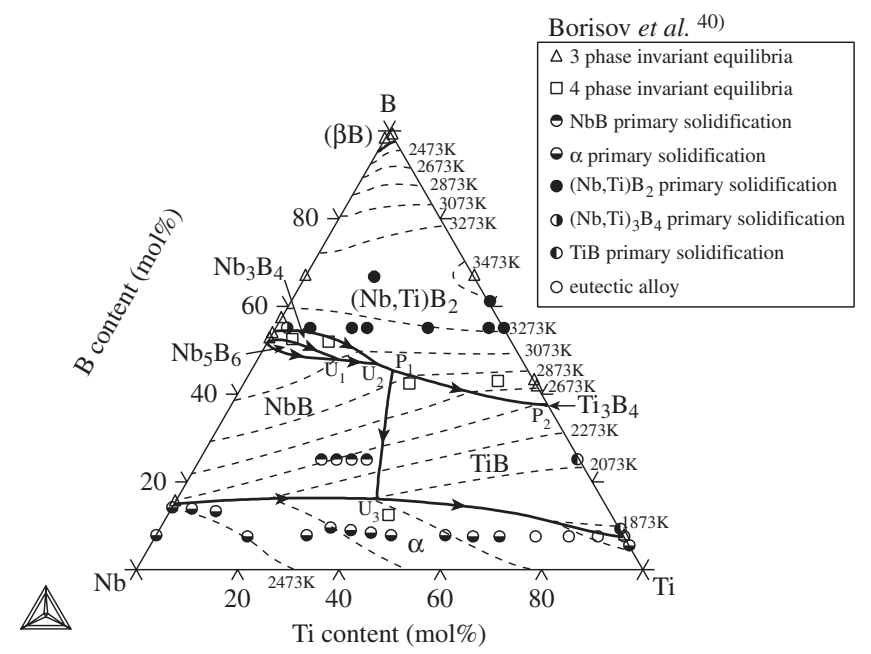

Fig. 11 The calculated liquidus projection of the $\mathrm{Nb}-\mathrm{Ti}-\mathrm{B}$ system.

(2) Due to a lack of experimental information on the thermodynamic properties of the $\mathrm{Nb}-\mathrm{Ti}-\mathrm{B}$ ternary system, the variation in the formation energies for the binary borides with a dissolving third component was evaluated for the $\mathrm{Nb}_{3} \mathrm{~B}_{2}, \mathrm{NbB}, \mathrm{TiB}, \mathrm{Nb}_{5} \mathrm{~B}_{6}$, $(\mathrm{Nb}, \mathrm{Ti})_{3} \mathrm{~B}_{4}$, and $(\mathrm{Nb}, \mathrm{Ti}) \mathrm{B}_{2}$ phases using first-principles calculations. Comparison of the calculated values with those obtained using the conventional CALPHAD approach showed considerable difference. The phase equilibria of the $\mathrm{Fe}-\mathrm{Nb}-\mathrm{B}$ ternary system were elucidated across the entire composition range by determining the thermodynamic functions based on the theoretical values as well as any available experimental information.

\section{Acknowledgment}

This work was partially supported by the Ministry of Education, Culture, Sports, Science and Technology, Grantin-Aid for Scientific Research on Priority Areas, "Atomic Scale Modification” (Area No. 424). 
Table 5 Invariant reaction points of the $\mathrm{Nb}-\mathrm{Ti}-\mathrm{B}$ ternary system.

\begin{tabular}{|c|c|c|c|c|c|}
\hline \multirow{2}{*}{ Type } & \multirow{2}{*}{ Invariant reaction } & \multicolumn{2}{|c|}{ Liquid composition } & \multirow{2}{*}{$\begin{array}{c}\text { Temperature } \\
\text { (K) }\end{array}$} & \multirow{2}{*}{ Reference } \\
\hline & & $(\mathrm{mol} \% \mathrm{Ti})$ & $(\mathrm{mol} \% \mathrm{~B})$ & & \\
\hline \multirow{3}{*}{$\mathrm{U}_{1}$} & \multirow{3}{*}{$\mathrm{L}+\mathrm{Nb}_{5} \mathrm{~B}_{6} \Leftrightarrow \mathrm{Nb}_{3} \mathrm{~B}_{4}+\mathrm{NbB}$} & 16.1 & 47.9 & 3068 & Present work \\
\hline & & 10.8 & 50.2 & 3070 & 41) \\
\hline & & $\sim 4$ & $\sim 53$ & $3068 \pm 16$ & 40) \\
\hline \multirow{3}{*}{$\mathrm{U}_{2}$} & \multirow{3}{*}{$\mathrm{L}+\mathrm{Nb}_{3} \mathrm{~B}_{4} \Leftrightarrow(\mathrm{Nb}, \mathrm{Ti}) \mathrm{B}_{2}+\mathrm{NbB}$} & 24.2 & 46.8 & 2978 & Present work \\
\hline & & 19.0 & 47.8 & 2966 & 41) \\
\hline & & $\sim 11$ & $\sim 53$ & $2965 \pm 17$ & 40) \\
\hline \multirow{3}{*}{$\mathrm{P}_{1}$} & \multirow{3}{*}{$\mathrm{L}+(\mathrm{Nb}, \mathrm{Ti}) \mathrm{B}_{2}+\mathrm{NbB} \Leftrightarrow \mathrm{TiB}$} & 28.1 & 45.3 & 2920 & Present work \\
\hline & & 22.0 & 46.4 & 2920 & 41) \\
\hline & & $\sim 33$ & $\sim 42$ & $2923 \pm 15$ & 40) \\
\hline \multirow{2}{*}{$\mathrm{P}_{2}$} & \multirow{2}{*}{$\mathrm{L}+(\mathrm{Nb}, \mathrm{Ti}) \mathrm{B}_{2}+\mathrm{TiB} \Leftrightarrow \mathrm{Ti}_{3} \mathrm{~B}_{4}$} & 62.1 & 37.5 & 2464 & Present work \\
\hline & & $\sim 52$ & $\sim 41$ & $2683 \pm 36$ & 40) \\
\hline \multirow{3}{*}{$\mathrm{U}_{3}$} & \multirow{3}{*}{$\mathrm{L}+\mathrm{NbB} \Leftrightarrow \mathrm{TiB}+\alpha$} & 39.5 & 16.1 & 2061 & Present work \\
\hline & & 49.6 & 14.9 & 2063 & 41) \\
\hline & & $\sim 39$ & $\sim 13$ & $2063 \pm 10$ & 40) \\
\hline
\end{tabular}

\section{REFERENCES}

1) N. Saunders and A. P. Miodownik: CALPHAD (Calculation of Phase Diagrams): A Comprehensive Guide, (Pergamon Materials Series, Elsevier Science Ltd, Oxford, 1998).

2) D. J. Singh: Planewaves, Pseudopotentials and the LAPW Method, (Kluwer Academic Pub., Boston, 1994).

3) P. Blaha, K. Schwarz, G. K. H. Madsen, D. Kvasnicka and J. Luiz: WIEN2k, An Augmented Plane Wave and Local Orbitals Program for Calculating Crystal Properties, (Karlheinz Schwarz, Technische Universität Wien, Austria, 2001).

4) J. P. Perdew, K. Burke and M. Ernzerhof: Phys. Rev. Lett. 77 (1996) 3865-3868.

5) O. Redlich and A. T. Kister: Ind. Eng. Chem. 40 (1948) 345-348.

6) K. Yoshitomi, Y. Nakama, H. Ohtani and M. Hasebe: ISIJ Int. 48 (2008) 841-850.

7) A. T. Dinsdale: CALPHAD 15 (1991) 317-425.

8) J. I. Murray: Phase Diagrams of Binary Titanium Alloys, (ASM, Metals Park, Ohio, 1987) pp. 188-194.

9) Y. Zhang, H. Liu and Z. Jin: CALPHAD 25 (2001) 305-317.

10) K. C. H. Kumar, P. Wollants and L. Delaey: CALPHAD 18 (1994) 7179.

11) T. Tokunaga, K. Terashima, H. Ohtani and M. Hasebe: Mater. Trans. 49 (2008) 2534-2540.

12) J. L. Murray, P. K. Liao and K. E. Spear: Phase Diagrams of Binary Titanium Alloys (ASM, Metals Park, Ohio, 1987) pp. 33-38.

13) P. Ehrlich: Z. Anorg. Chem. 259 (1949) 1-41.

14) J. T. Norton, H. Blumenthal and S. J. Sindeband: Metall. Trans. 185 (1949) 749-751.

15) A. E. Palty, H. Margolin and J. P. Nielsen: Trans. ASM 46 (1954) 312 328.

16) R. G. Fenish: NRM-138 (1964) 1-37.

17) E. Rudy and S. Windisch: Ternary Phase Equilibria in Transition Metal-Boron-Carbon-Silicon System. Part I, (Related Binary System, Ti-B System. vol. VII) Technical Report No. AFML-TR-65-2, Part I, vol. VII, (1966).

18) K. E. Spear, P. McDowell and F. McMahon: J. Am. Ceram. Soc. 69 (1986) C4-C5.

19) R. G. Fenish: Trans. AIME 236 (1966) 804.

20) L. Brewer and H. Haraldsen: J. Electrochem. Soc. 102 (1955) 399-406.

21) C. E. Lowell and W. S. Williams: Rev. Sci. Instrum. 32 (1961) 1120 1123.

22) W. Williams: J. Phys. Chem. 65 (1961) 2213-2216.

23) P. Schissel and O. Trulson: J. Phys. Chem. 66 (1962) 1492-1496.

24) V. V. Fesenko and A. S. Bolgar: Soviet Powder Metall. Metal. Ceram. 1
(1971) 11.

25) G. M. Kibler, T. F. Lyon, M. I. Linevsky and V. J. Desantis: Tech. Rep. No. WADD-TR-60-646, Part III, Vol. 2, (General Electric Co., Evansdale, Ohio, 1964).

26) E. J. Huber: J. Chem. Eng. Data 11 (1966) 430-431.

27) D. R. Stull and H. Prophet: JANAF Thermochemical Tables, 2nd ed., (Nat. Stand. Ref. Data Ser., Nat. Bur. Stand., USA, 1971) p. 37.

28) R. Hultgren, P. D. Desai, D. T. Hawkins, M. Gleiser and K. K. Kelley: Selected Values of the Thermodynamic Properties of Binary Alloys, (ASM, Metals Park, Ohio, 1973) pp. 344-349.

29) V. V. Akhcchinskij and N. A. Chirin: Thermodynamics of Nuclear Materials 1974, Vol. II, (IAEC, Vienna, 1975) pp. 467-476.

30) T. J. Yurick and K. E. Spear: Thermodynamics of Nuclear Materials 1979, Vol. I, (IAEA-SM-236/53, Vienna, 1980) pp. 73-90.

31) B. Walker, C. Ewing and R. Miller: J. Phys. Chem. 61 (1957) 16821683.

32) A. N. Krestovnikov and M. S. Vendrekh: Izv. V. U. Z. Tsvetn. Metall. 2 (1959) 54-57.

33) R. Mezaki, E. Tilleux, D. W. Barnes and J. L. Margrave: Thermodynamics of Nuclear Materials 1962, (IAEA, Vienna, 1962) pp. 775-788.

34) R. A. McDonald, F. D. Oetting and H. Prophet: Rep. N64-18824, (Dow Chemical Co., Midland, Michigan, 1963).

$35)$ D. S. Neel, C. D. Pears and S. Oglesby: Tech. Doc. Rep. No. ASD-TDR62-765, (Southern Res. Inst., Birmingham, Alabama, 1963).

36) V. A. Kirilin, A. E. Sheindlin, V. Ya. Chekhovskoi and V. I. Tyukaev: Teplofiz. Vys. Temp. 2 (1964) 710-715.

37) P. Villars: Pearson's Handbook, Desk Edition, Crystallographic Data for Intermetallic Phases, Vol. 1 (ASM International, Materials Park, Ohio, 1997).

38) M. W. Chase: NIST-JANAF Thermochemical Tables, 4th ed., (American Institute of Physics for the National Institute of Standards and Technology, 1998).

39) Yu. B. Kuz'ma: Soviet Powder Metall. Metal. Ceram. 10 (1971) 298300.

40) D. B. Borisov, L. V. Artyukh, A. A. Bondar, P. S. Martsenyuk, A. V. Samelyuk, N. I. Tsiganenko, O. S. Fomichov and T. Ya. Velikanova: Powder Metall. Metal. Ceram. 46 (2007) 58-71.

$41)$ V. T. Witusiewicz, A. A. Bondar, U. Hecht, S. Rex and T. Ya. Velikanova: J. Alloy. Compd. 456 (2008) 143-150.

42) Z. W. Lu, S.-H. Wei, A. Zunger, S. Frota-Pessoa and L. G. Ferreira: Phys. Rev. B 44 (1991) 512-544.

43) A. van de Walle and G. Ceder: J. Phase Equil. 23 (2002) 348-359.

44) F. D. Murnaghan: Proc. Nat. Acad. Sci. USA 30 (1944) 244-247.

$45)$ V. T. Witusiewicz, A. A. Bondar, U. Hecht, S. Rex and T. Ya. Velikanova: J. Alloy. Compd. 448 (2008) 185-194. 